\title{
MS11-P02 | HARNESSING THE DATA: INTEGRATING DATA FLOWS ACROSS HOME \\ LABORATORIES, FACILITIES AND DATA REPOSITORIES IN PROTEIN CRYSTALLOGRAPHY
}

Marquez, Jose Antonio (EMBL, Grenoble, Grenoble, FRA)

The systematic introduction of automation in structural biology over the last decades has enabled the study of ever more challenging targets. However, it has brought new challenges, concerning data management and integration. Typically, crystallography experiments are carried out across multiple sites including home laboratories and synchrotron facilities and spans relatively long periods of time before data is deposited in one of the worldwide Protein Data Bank sites. While some of these facilities use data management systems they are not interconnected. Moreover, new applications, like fragment screening and serial, crystallography, producing large amounts of data in a short time are becoming mainstream. To address these problems, we have developed the Crystallography Information Management System, CRIMS. A web-based software suit enabling automated sample tracking along the experimental crystallography workflow, from pure protein to model refinement. This system provides interfaces for crystallization screening and optimization that can be connected to the most popular crystallization robots, can establish communication with multiple synchrotron facilities, exchanging unique sample identifiers to support both manual and automated data collection and provides for the first time, automated, uninterrupted sample tracking along the whole process. Recently, we have incorporated communication with automated data processing pipelines to support large scale fragment screening. The CRIMS system is installed in multiple laboratories in Europe and is being used by over 1500 scientists. The experience on the use of this system and its potential to support automated data tracking and deposition and foster the development of an European Open Science Cloud will be discussed. 